Bull. Mater. Sci., Vol. 15, No. 1, February 1992, pp. 27-34. (C) Printed in India.

\title{
Dynamic fracture mechanics-A scientific tool for the prevention of catastrophic failure
}

\author{
R KRISHNAKUMAR \\ Department of Mechanical Engineering, Indian Institute of Technology, Madras 600036, \\ India
}

\begin{abstract}
The major area of research in dynamic fracture has been the extension of the concept of static fracture toughness to predict crack arrest for a propagating crack. In this work crack propagation due to a ductile (microvoid) mechanism and cleavage (brittle) mechanism, as well as transition from one mode to another, has been analysed theoretically. Dynamic fracture toughness as a function of crack velocity has been determined. Temperature distribution near a propagating crack tip has been predicted for plane stress condition. The effect of reflected stress wave in a single edge notch specimen under transient crack growth conditions has also been analysed.
\end{abstract}

Keywords. Dynamic fracture; crack propagation; stress wave catastrophic failure.

\section{Introduction}

Dynamic fracture is concerned with problems in which inertial resistance of the material is important. There are basically two classes of such problems. The first one deals with a stationary crack subjected to a time-varying load. The second problem is that of a rapidly propagating crack under a stationary or time-varying load. In the former case, one would like to determine the conditions under which the crack would initiate while in the latter problem, attention is focussed on determining a criterion for continuing the crack growth. This paper deals with rapidly propagating cracks.

Rapid crack propagation can take place either under steady state or transient conditions. Steady-state crack growth is one in which an observer, travelling with the crack tip, sees no change in field quantities around the tip. Dynamic fracture has a significant role to play in a number of areas (Freund 1986). Rapid crack propagation and arrest in pressure vessels and piping systems, cleavage fracture of crystalline materials, structures subjected to impact loading are some of the dynamic fracture mechanics application areas.

For a propagating crack with a velocity $v$, which is assumed to be less than any characteristic wave speed of the material Freund and Clifton (1974) and Nilsson (1974) showed that the components of stress can be expressed as:

$$
\sigma_{i j}=\lim _{r \rightarrow 0} \frac{K_{I}(t)}{\sqrt{2 \pi r}} \Sigma_{i j}\left(v / c_{1}, v / c_{2}, \theta\right)
$$

where $(r, \theta)$ indicates a polar coordinate system with the origin at the crack tip. $K_{I}(t)$ is a time-dependent dynamic stress intensity factor and $\Sigma_{i j}$ is a universal function dependent on $\theta$, crack speed $v$, longitudinal wave speed $c_{1}$ and shear wave speed $c_{2}$. Equation (1) underlines the similarity in the role played by dynamic stress intensity factor and static stress intensity factor. As an extension of the ideas of static condition, 
a dynamic fracture criterion for mode I small scale yielding is postulated as

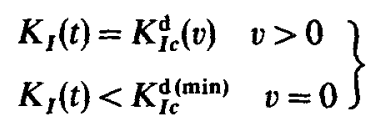

$K_{I c}^{\mathrm{d}}$ is called the dynamic fracture toughness of the material, which is a function of the crack speed and material properties.

Another factor, apart from the material inertia which affects the dynamic fracture toughness, is the rate sensitivity of the material. The strain rates experienced by material elements close to the crack tip are generally very large, ranging from $10^{5} \mathrm{~s}^{-1}$ to $10^{6} \mathrm{~s}^{-1}$ for typical crack velocities (Freund and Hutchinson 1985). At such high strain rates, many metals display sharp increase in flow stress over slow loading value. The effect of this elevation in flow stress is expected to be vastly different for fracture by cleavage as compared to fracture by a micro-void mechanism.

\section{Dynamic growth of tensile cracks}

Finite element analysis of steady-state dynamic crack growth under mode I, plane strain small-scale yielding condition, has been performed (Krishna Kumar 1990). A rate-dependent plastic material (to take into account the material rate sensitivity) characterized by the over-stress model (Perzyna 1966) has been used for the analysis. A modified version of the rate tangent modulus method (Pierce et al 1984) is used to update the stresses. The main objective of this study is to obtain theoretically the dependence of dynamic fracture toughness on crack speed. Crack propagation due to a ductile (micro-void) mechanism or a brittle (cleavage) mechanism, as well as transition from one mode to another are considered. In order to determine the fracture toughness for ductile mode of crack growth, a plastic strain based criterion has been used. For mode I, plane strain conditions, intense plastic strain concentration occurs above and below the crack tip. Under these conditions, it is required that for continuing crack growth the equivalent plastic strain at a small (material) characteristic distance $X_{d}$ directly above (or below) the crack tip must be equal to a critical value $Y_{d}$. Two values of critical plastic strains namely, $2 \varepsilon_{0}$ and $5 \varepsilon_{0}$ have been chosen for the analysis.

A simple stress-based fracture criterion is adopted to model crack initiation and growth by a brittle cleavage mechanism. This requires that the opening stress $\sigma_{22}$ should be equal to a critical value at a small characteristic distance $X_{b}$ directly ahead of the crack tip (Ritchie et al 1973). In the present work, in order to have a common basis for expressing the results, it is assumed that $X_{b}=2 X_{d}$. Also the critical stress $\sigma_{b}$ is taken as $3 \cdot 5 \sigma_{0}$.

In order to obtain the fracture toughness versus crack speed relationships for a given set of material constants, a new parameter $\hat{\beta}$ is introduced as:

$$
\widehat{\beta}=E \beta x_{d} / \sigma_{0} c_{s}=\beta^{*} m_{s} \hat{x}_{d} .
$$

In figure 1 , the estimated ratio $K / K_{c}$, where $K$ indicates a dynamic fracture toughness and $K_{c}$ the static fracture toughness, is plotted against crack speed for a value of critical plastic strains of $5 \varepsilon_{0}$ and a rate exponent, $n$ of $4 \cdot 0$. Results are displayed for $\hat{\beta}$ ranging from 0.02 to 1.0 . This range of values for $\hat{\beta}$ is typical for structural metals. From these figures, it can be observed that materials with a higher degree of rate sensitivity (smaller $\hat{\beta}$ ) have higher dynamic fracture toughness, where crack advance 


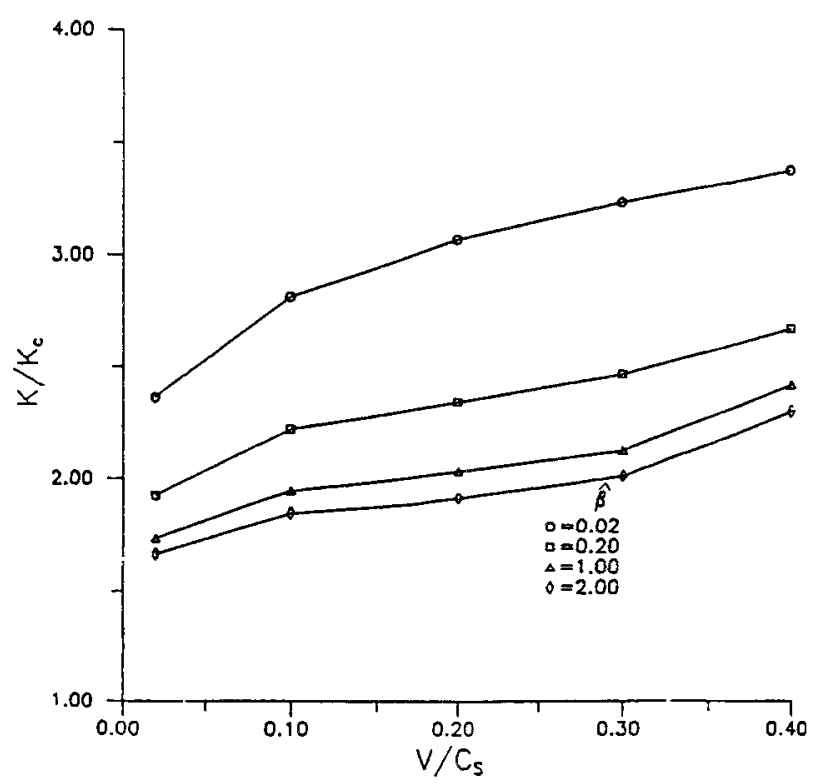

Figure 1. Variation of $K / K_{c}$ with normalized crack velocity ductile mode of failure. $n=4 . \gamma_{d}=5 \cdot 0 \varepsilon_{0}$. Plane strain.

occurs by a ductile mechanism. Also, for small $\hat{\beta}$, the increase in $K / K_{c}$ with crack speed is quite strong when crack speeds are very small and inertial effects are unimportant.

The variation of $K / K_{c}$ for cleavage fracture initiation and growth is shown in figure 2. It can be seen from this figure that for cleavage crack growth, the dynamic fracture toughness corresponding to any value of $\hat{\beta}$ decreases initially with crack speed, reaches a minimum and then increases for large speeds. Also, $K / K_{c}$ is higher for larger $\hat{\beta}$ which corresponds to a lower degree of rate sensitivity. This may be due to computing roles played by material rate sensitivity and material inertial (Krishna Kumar 1990).

It has long been recognized that a crack may initiate by a ductile mechanism but subsequently propagate in either a ductile or brittle mode. This aspect has received very little attention using analytical methods. The results obtained from this study are shown in figure 3 . The results demonstrate that, following initiation, a crack will accelerate from zero velocity under increasing applied $K$ with local separation occurring by a ductile mechanism. Upon reaching the transition point, a cleavage mode would become operative, resulting in very large speeds. The results obtained are in general agreement with microstructural observations of mode conversions during fracture initiation.

Steady-state, dynamic crack growth has also been analysed under mode I plane stress small-scale yielding conditions using a finite element procedure. A Perzyna type, overstress viscoplastic constitutive equation, similar to that used in the first part, has been employed in this analysis. A fracture criterion based on critical plastic strain has been imposed to obtain (theoretically) the variation of dynamic fracture toughness with crack speed, when crack propagation occurs in a ductile mode. The results are presented in figures 4 and 5 . The results obtained are similar to that 


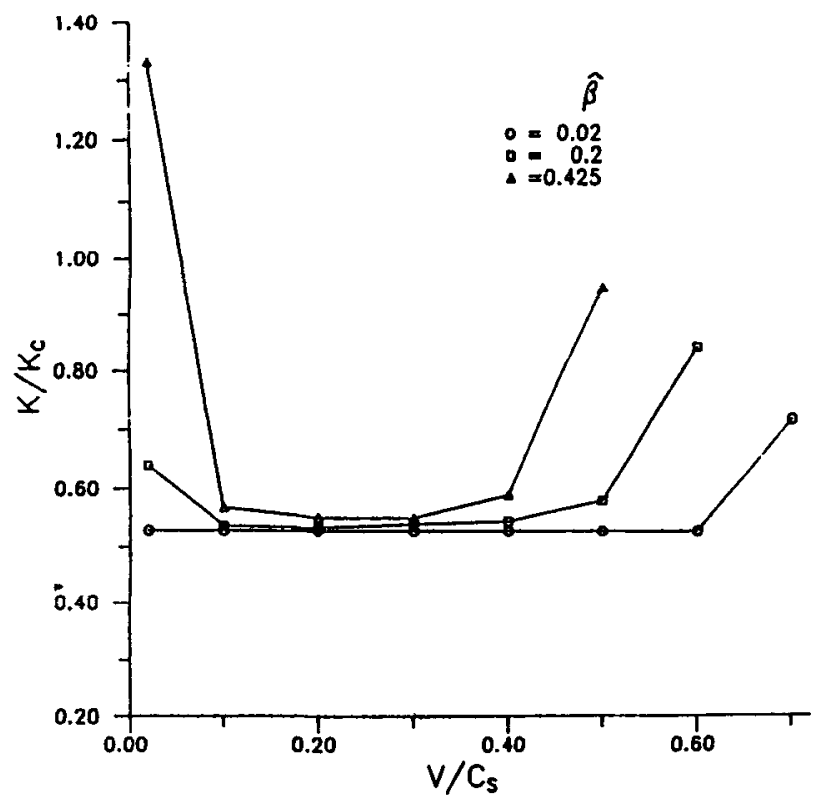

Figure 2. Variation of $K / K_{c}$ with normalized crack velocity cleavage mode of failure. $n=2 . \sigma_{22}=3 \cdot 5 \sigma_{0}$. Plane strain.

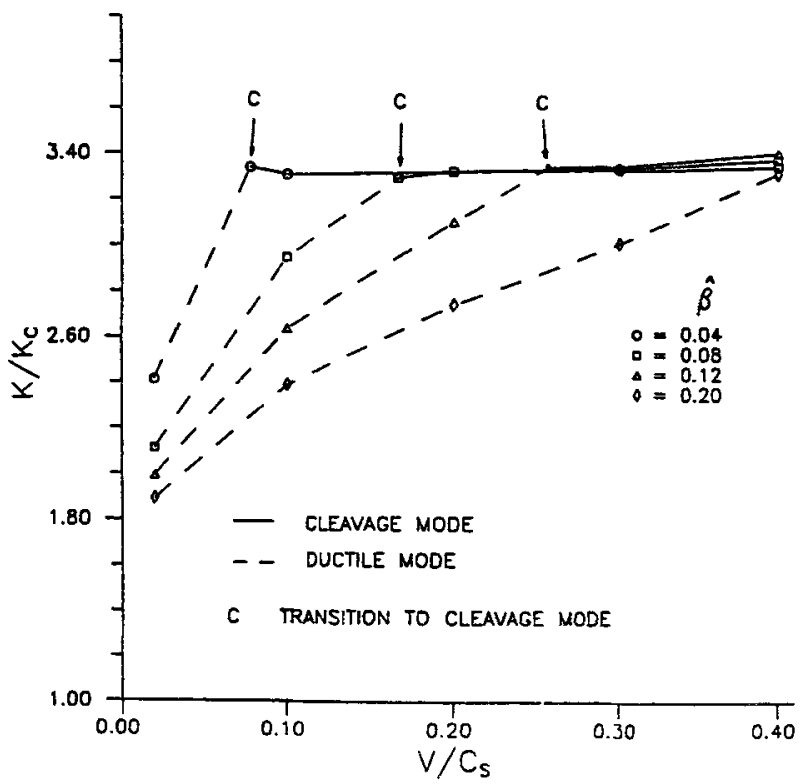

Figure 3. Variation of $K / K_{\mathrm{c}}$ with normalized crack velocity ductile cleavage mode transition. $n=2$. Plane strain.

obtained for plane strain conditions. It may be concluded from these studies that cleavage cracks may get arrested abruptly, whereas ductile crack arrest may be more gradual.

The temperature rise near a propagating crack tip has an important role to play 


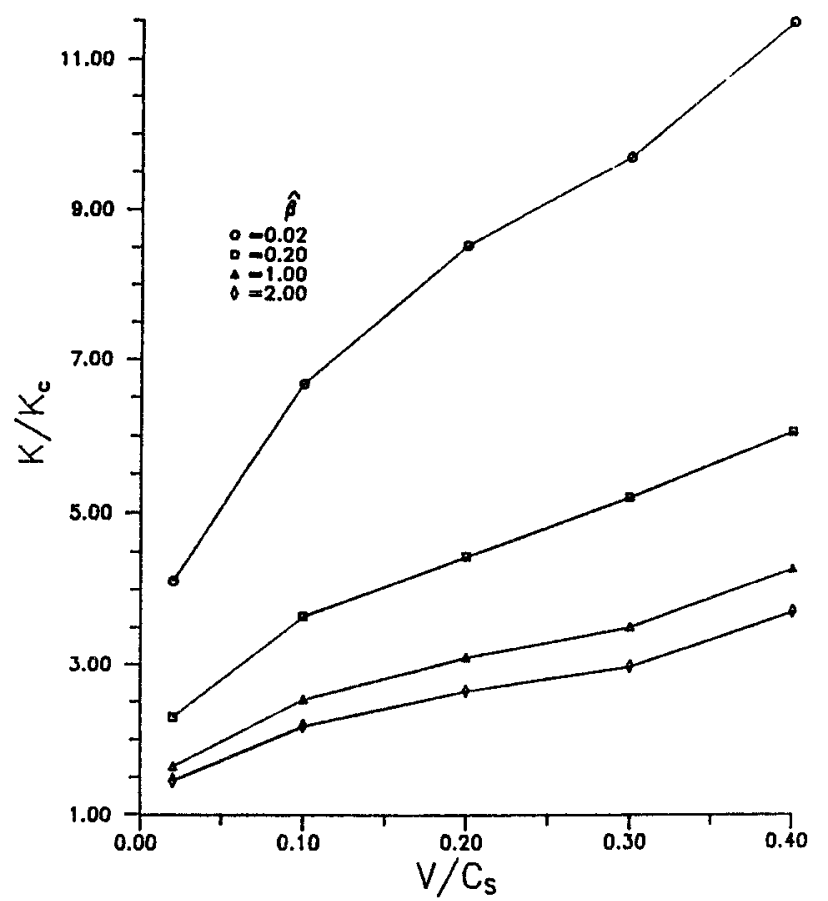

Figure 4. Variation of $K / K_{c}$ with normalized crack velocity. $n=2 . \gamma_{d}=5 \cdot 0 \varepsilon_{0}$. Plane stress.

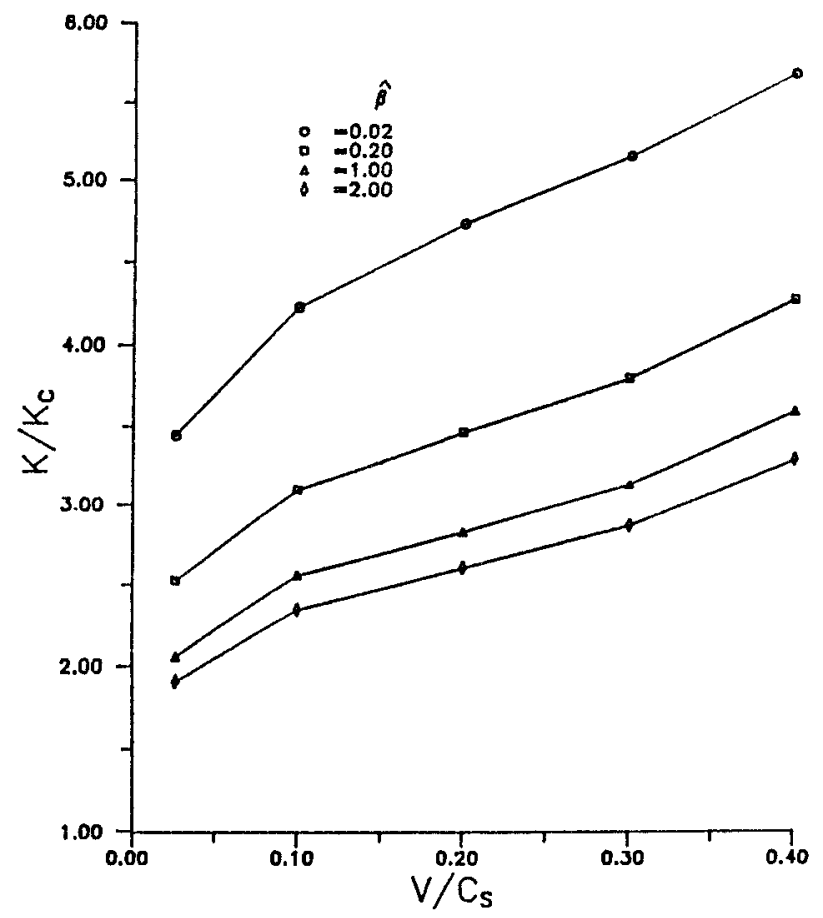

Figure 5. Variation of $K / K_{c}$ with normalized crack velocity $n=4 . \gamma_{d}=5 \cdot 0 \varepsilon_{0}$. Plane stress. 
in determining the material behaviour during rapid crack growth. The viscoplastic work rate calculated with mode I plane stress small-scale yielding condition has been used as heat input to determine the temperature rise by solving the governing conduction/convection equation also by a finite element method. The stream-line upwinding Petrov-Galerkin formulation (Brooks and Hughes 1982) has been employed for this purpose because of the high P'eclet number that results in such types of analysis. A parameter $\alpha$ consisting of material constants and stress intensity factor $K$ has been introduced. This parameter is independent of crack velocity. Properties used in temperature calculations correspond to that of a high strength cold-rolled steel. The effect of strain rate sensitivity and crack speed on the temperature distribution near the crack tip is examined. The results so obtained showed that the temperature rise is highest for low crack speeds, when the parameter assumes a small value. However, for larger $\alpha$ values it is found that the temperature rise immediately in front of the crack tip, at a normalized distance, initially increases with crack speed and then decreases (figures 6-7).

Energy flow to the crack tip during dynamic crack growth under transient conditions is considered in the final part of the study. From a practical standpoint, it is important to understand the effects of stress waves reflected from the specimen boundaries on the energy flow to the crack tip and the near tip fields in general. This issue has been pointed out in the experimental studies of a number of investigators. An explicit dynamic finite element method along with a nodal release algorithm (Rydholm et al 1978) has been used to simulate the crack growth. A modified rate tangent modulus method has been used to update the stresses.

The cracks were allowed to grow at different velocities in a single edge notcin (SEN) specimen and the energy flow to the crack tip was calculated as a function of crack length. It was found that the energy flow $\gamma$, during the initial phase of crack growth increases almost linearly with the crack length and exhibits a great sensitivity to crack

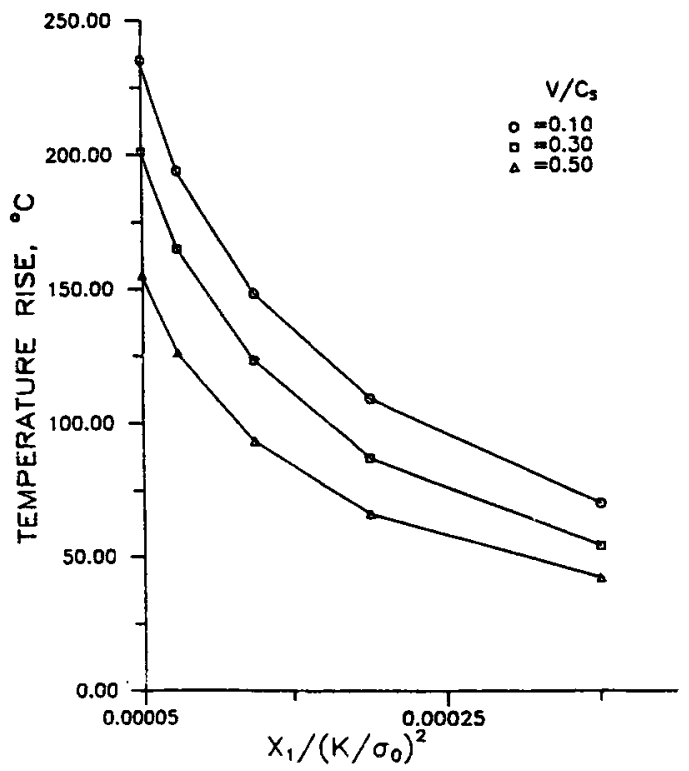

Figure 6. Temperature rise ahead of the crack. $\alpha=2 \cdot 5$. Plane stress. 


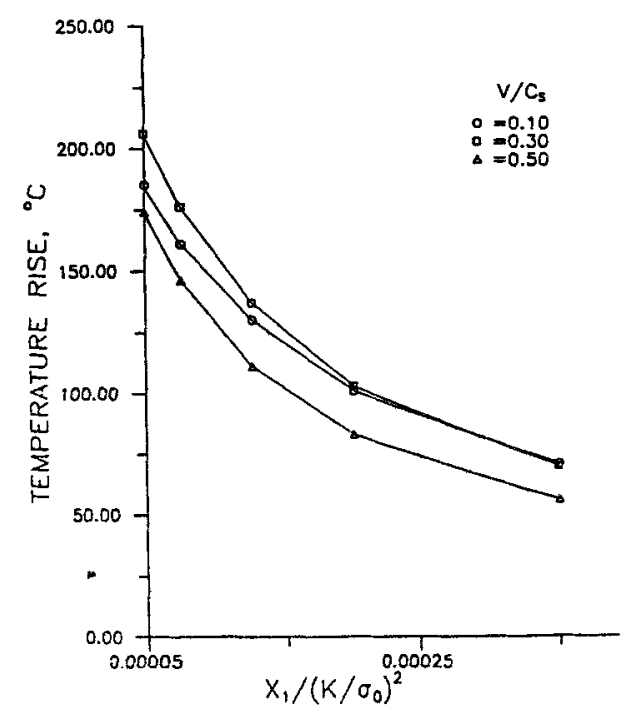

Figure 7. Temperature rise ahead of the crack. $\alpha=12 \cdot 5$. Plane stress.

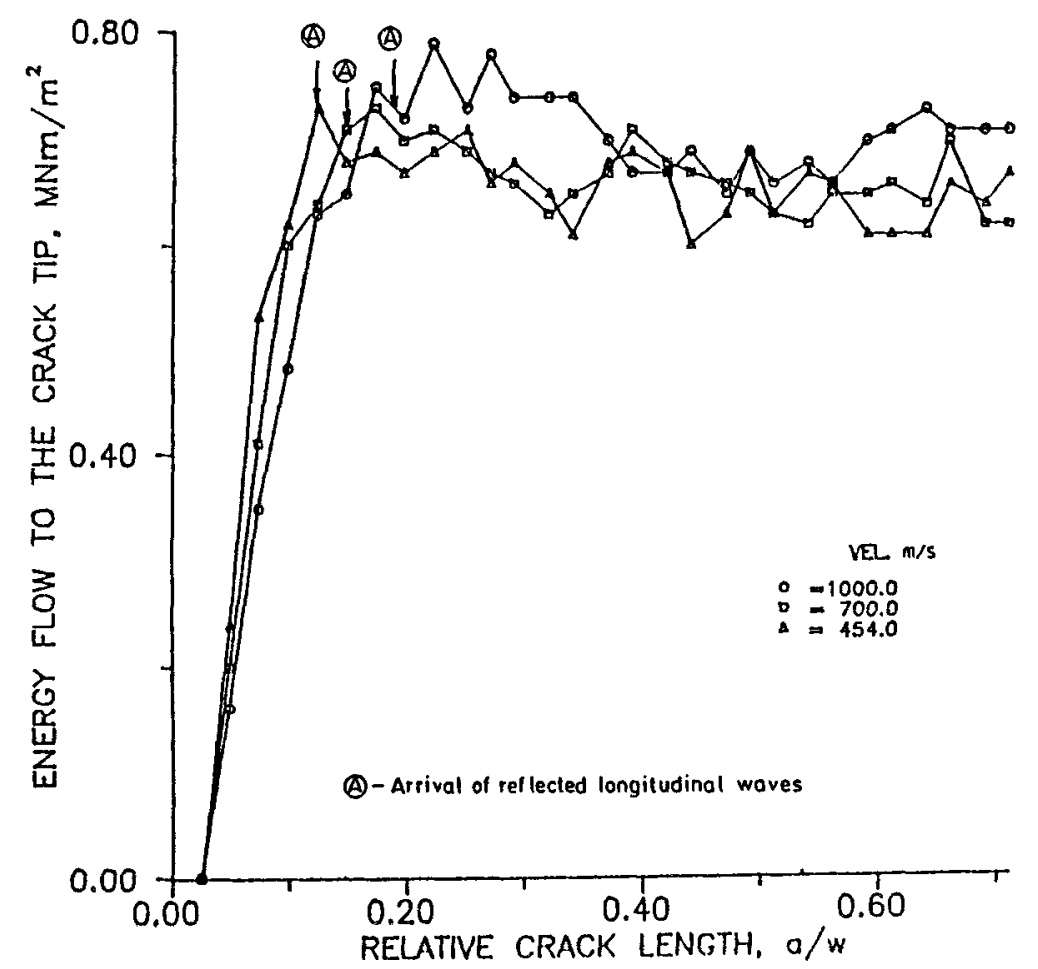

Figure 8. Energy flow to the crack tip as the crack propagates at different velocities.

speed, but on arrival of the longitudinal waves reflected from the specimen boundaries, begins to oscillate about a constant value (figure 8). Also, the energy flow is quite insensitive to crack velocity at large crack lengths. These factors may have an important effect on the suitability of specimen configurations like SEN and double 
cantilever beam (DCB) to obtain dynamic fracture toughness versus crack velocity for metals.

\section{Conclusions}

The study of rapid crack growth and arrest is challenging to both experimentalists and theoreticians. But the technological implications in understanding this phenomenon are immense. This decade is certain to witness rapid strides in dynamic fracture mechanics.

\section{Acknowledgements}

The author wishes to thank Dr R Narasimhan, Department of Mechanical Engineering, Indian Institute of Science, Bangalore for his immense help during the course of this study.

\section{References}

Brooks A N and Hughes T J R 1982 Comp. Math. App. Mech. Eng. 32199

Freund L B 1986 Tenth U.S. Congress of Applied Mechanics, Austin, Texas

Freund L B and Clifton R J 1974 J. Elasticity 4293

Freund L B and Hutchinson J W 1985 J. Mech. Phys. Solids 33169

Krishna Kumar R 1990 Finite element analysis of transient and steady state dynamic fracture in a viscoplastic material, PhD Thesis, Indian Institute of Technology, Madras

Nilsson F 1974 J. Elasticity 493

Perzyna P 1966 in Advances in Applied Mechanics (New York: Academic Press) 243

Pierce C, Shih C F and Needleman A 1984 Computers \& Structures 18875

Ritchie R O, Knott J E and Rice J R 1973 J. Mech. Phys. Solids 21395

Rydholm G, Fredrikson B and Nilsson F 1978 in Numerical Methods in Fracture (eds) A R Luxmore and D R J Owen (Swansea: Univ. College) 